\title{
Analytical robustness of quantitative NIR chemical imaging for Islamic paper characterization
}

\author{
Hend Mahgoub*a, John R. Gilchrist ${ }^{\mathrm{b}}$, Thomas Fearn ${ }^{\mathrm{c}}$, Matija Strlič ${ }^{\mathrm{a}}$ \\ ${ }^{a}$ Institute for Sustainable Heritage, University College London, London, UK; ${ }^{\mathrm{b}}$ Gilden Photonics Ltd, \\ Glasgow, UK; ' Department of Statistical Science, University College London, London, UK \\ *hend.mahgoub.13@ucl.ac.uk
}

\begin{abstract}
Recently, spectral imaging techniques such as Multispectral (MSI) and Hyperspectral Imaging (HSI) have gained importance in the field of heritage conservation. This paper explores the analytical robustness of quantitative chemical imaging for Islamic paper characterization by focusing on the effect of different measurement and processing parameters, i.e. acquisition conditions and calibration on the accuracy of the collected spectral data. This will provide a better understanding of the technique that can provide a measure of change in collections through imaging.
\end{abstract}

For the quantitative model, special calibration target was devised using 105 samples from a well-characterized reference Islamic paper collection. Two material properties were of interest: starch sizing and cellulose degree of polymerization (DP). Multivariate data analysis methods were used to develop discrimination and regression models which were used as an evaluation methodology for the metrology of quantitative NIR chemical imaging.

Spectral data were collected using a pushbroom HSI scanner (Gilden Photonics Ltd) in the 1000-2500 nm range with a spectral resolution of $6.3 \mathrm{~nm}$ using a mirror scanning setup and halogen illumination. Data were acquired at different measurement conditions and acquisition parameters.

Preliminary results showed the potential of the evaluation methodology to show that measurement parameters such as the use of different lenses and different scanning backgrounds may not have a great influence on the quantitative results. Moreover, the evaluation methodology allowed for the selection of the best pre-treatment method to be applied to the data.

Keywords: NIR, Spectral imaging, Quantitative analysis, Pushbroom scanner, Calibration, Islamic paper

\section{INTRODUCTION}

Heritage objects are well-known for their compositional inhomogeneity due to materials and processes used in their production ${ }^{1}$. Techniques such as hyperspectral imaging have combined imaging and material characterization ${ }^{2}$ by expanding spectroscopic applications from spot analysis to the examination of the entire surface of an object which consequently provided valuable information about the distribution of material properties ${ }^{3-7}$. The spectral data collected especially in the near infrared region could be used to study the physical and chemical composition of objects ${ }^{8-10}$.

Quantitative investigation is a complex process due to the lack of standard materials for calibration which should be representative of the complexity of the real objects. Thus, most of the focus of the spectral imaging techniques was focusing on qualitative applications.

Similar to the spectroscopic applications, multivariate data analysis methods are required due to the complexity of the collected spectral data that consist of two dimensions for spatial information and one dimension for spectral information ${ }^{6,9}$.

In this paper, the limitations and benefits of quantitative chemical imaging and its analytical robustness will be studied by mapping the chemical composition and condition of Islamic paper in particular. Cellulose degree of polymerization (DP), in addition to one of the main characteristic components of Islamic paper ${ }^{11}$ : starch sizing, were of interest. An evaluation methodology will be developed to provide better understanding of the technique using discrimination and

Optics for Arts, Architecture, and Archaeology VI, edited by Luca Pezzati,

Piotr Targowski, Proc. of SPIE Vol. 10331, 103310P - (C) 2017 SPIE

CCC code: $0277-786 X / 17 / \$ 18 \cdot$ doi: $10.1117 / 12.2271971$

Proc. of SPIE Vol. 10331 103310P-1 
regression models for the calibration of the two properties. To build these quantitative models, a special calibration target was devised from the well-characterized reference Islamic paper collection of UCL Institute for Sustainable Heritage.

Spectral data were acquired using a pushbroom scanner from Gilden Photonics in the 1000-2500 nm range using a mirror scanning setup and illuminated with a line of halogen lamps.

Different measurement conditions and acquisition parameters may have an effect on the accuracy of the collected spectra such as the spectral power distribution of illumination and its intensity, optics and scanning backgrounds. Even post processing methods used in the data analysis stage could affect the quantitative results. In view of this, we studied the metrology of quantitative chemical mapping based on hyperspectral imaging in the NIR region using the developed evaluation methodology.

\section{METHODOLOGY}

\subsection{Hyperspectral Imaging (HSI) System and Acquisition Parameters}

Spectral data in the range 1000-2500 $\mathrm{nm}$ was collected using a pushbroom HSI scanner manufactured by Gilden Photonics Ltd (Figure 1). The scanner consists of a line-spectrograph (Specim, ImSpector N25E) with a 30- $\mu \mathrm{m}$ slit connected to a Mercury-Cadmium-Telluride (MCT) camera and a mirror scanning setup. The system includes a camera with a maximum frame rate of $100 \mathrm{fps}$, a fixed scanning distance $\sim 110 \mathrm{~cm}$ and a spectral resolution of $6.3 \mathrm{~nm}$. Two different lenses were also used in this research to acquire the data; $30 \mathrm{~mm}$ and $56 \mathrm{~mm}$. The illumination is done using a line of halogen lamps at an angle of $\sim 30^{\circ}$ and a distance of $\sim 18 \mathrm{~cm}$. The illumination was used with two intensities; 500 and $250 \mathrm{~W}$. During all the experiments, the lights were warmed up for $\sim 1 \mathrm{~h}$ before scanning for stabilization. Different combinations of acquisition parameters were used to collect the spectral measurements which are listed in Table 1.

Table 1. Hyperspectral imaging scanner different combinations of acquisition parameters used in this study.

\begin{tabular}{|l|l|l|l|}
\hline & Set 1 & Set 2 & Set 3 \\
\hline Lens & $30 \mathrm{~mm}$ & $30 \mathrm{~mm}$ & $56 \mathrm{~mm}$ \\
\hline Exposure & $6 \mathrm{~ms}$ & $9.4 \mathrm{~ms}$ & $7 \mathrm{~ms}$ \\
\hline Scan speed & $54.9 \mathrm{~mm} / \mathrm{s}$ & $51 \mathrm{~mm} / \mathrm{s}$ & $29 \mathrm{~mm} / \mathrm{s}$ \\
\hline Lights & $500 \mathrm{~W}$ & $250 \mathrm{~W}$ & $500 \mathrm{~W}$ \\
\hline Aperture & $\mathrm{F} / 2.0$ & $\mathrm{~F} / 2.0$ & $\mathrm{~F} / 4.0$ \\
\hline
\end{tabular}

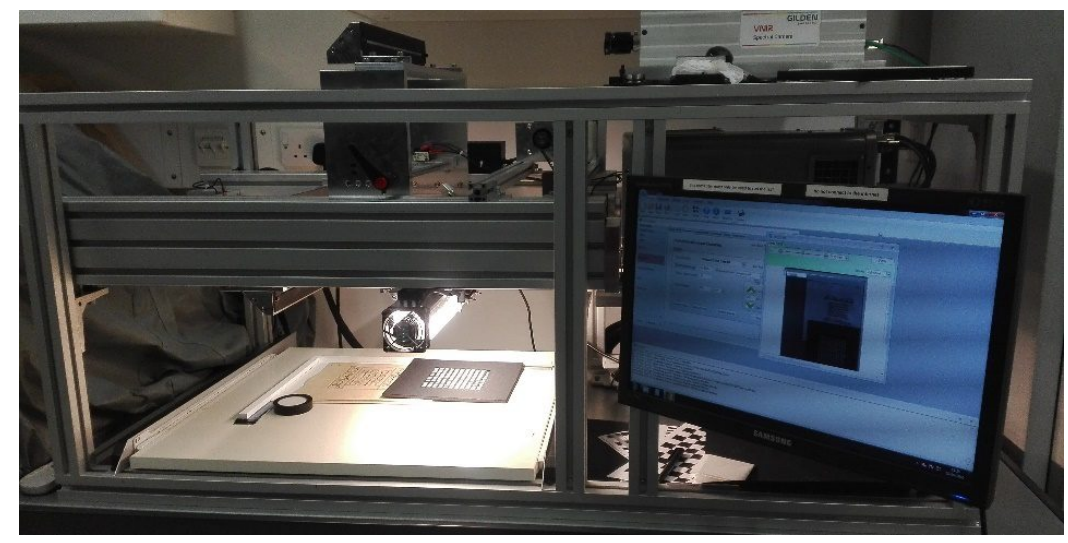

Figure 1. Hyperspectral imaging scanner used in this study. 


\subsection{Calibration Target Development}

105 samples were selected from the well-characterized reference collection of Islamic paper to devise the calibration target (Figure 2). The collection is part of a substantial collection of 228 papers belongs to UCL Institute for Sustainable Heritage (UCL-ISH). It was collected from different sources in Central Asia, Near East and North Africa $\left(15^{\text {th }}-19^{\text {th }}\right.$ century) for the purpose of material analysis ${ }^{11}$.

The identification of starch and DP of cellulose in paper of the samples were analyzed using chemical analytical methods $^{11}$ : iodine test ${ }^{12,13}$ and viscometric standard method with Mark-Houwink-Sakurada equation ${ }^{14,15}$, respectively.

Starch was identified in 45 samples versus 60 samples without starch. However, DP measurements were conducted for only 57 samples as the other samples contained lignin, which cannot be measured using the traditional analytical method. The average DP of all the samples is 848 with a standard deviation of 427.

The design of the target permits the use of different backgrounds during scanning as seen in Figure 2 (right).
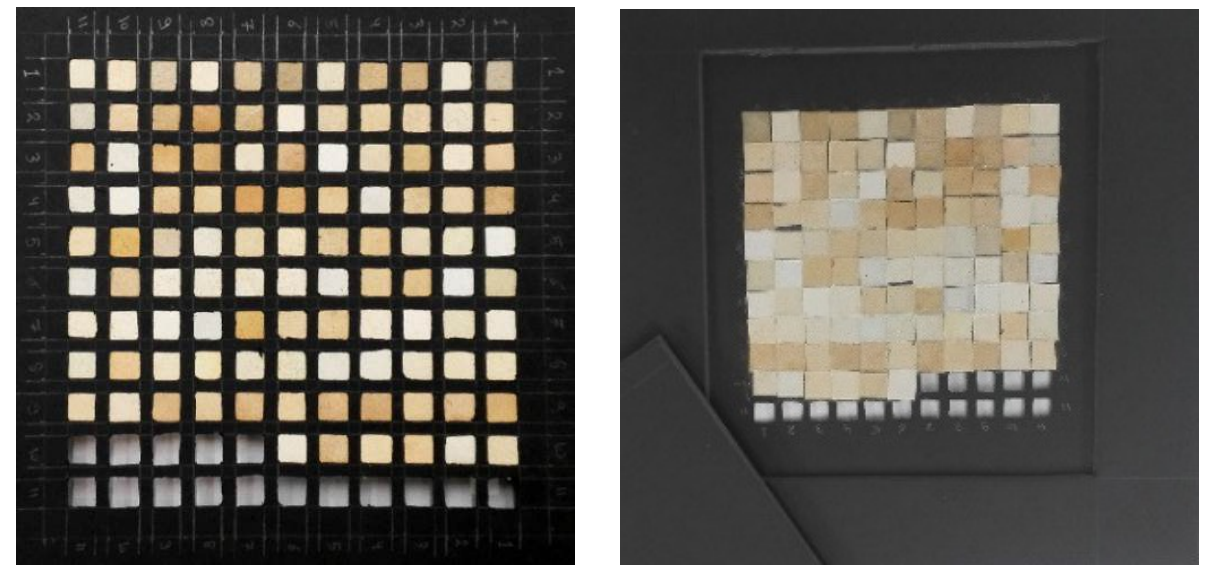

Figure 2. Calibration target with 105 well-characterized samples of Islamic paper (front - left, and back - right sides).

\subsection{Multivariate Data Analysis (MVA)}

All spectral data was acquired in the RAW format, then spectrally and spatially corrected using the manufacturer's software (SpectraSENS). Spectralon reference standard and detector dark current were used in the correction process.

Using Matlab (R2015b) with PLS-Toolbox library (Eigenvector), different pre-treatments and multivariate data analysis methods were used to select best practice for the collected data.

In this study, three different spectral pre-treatments ${ }^{16}$ were tested to optimize the quality of the results; Standard Normal Variate (SNV), Multiplicative Scatter Correction (MSC) and Savitzky-Golay (SG) filtering methods. This was followed by either a discriminant or a regression method.

For the discrimination of starch presence, principal component analysis (PCA) was used on the spectra to reduce variability followed by a linear discriminant (LDA) on the selected PC scores - PCDA method ${ }^{17-20}$ was used with the spectral data of all the samples in the Islamic paper target.

Partial least squares (PLS) regression method ${ }^{1820-22}$ was used to build the calibration model for the determination of DP for unknown samples using 57 samples from the Islamic paper target and the actual DP measurements.

Leave one out cross-validation (LOOCV) was used to validate the results of the calibration models. Evaluation of the DP regression model is based on the root mean square error of the cross-validation (RMSECV) and the correlation coefficient $(\mathrm{Rcv})^{18}$. On the other hand, the proportion of correctly identified samples (\% Correctness) was used to validate the accuracy of the starch discrimination model. 


\section{RESULTS}

\subsection{Discrimination Model (Starch Presence)}

As starch sizing is one of the main characteristic of Islamic paper, a discrimination calibration model was developed to identify the presence of starch in samples based on the calibration target data. Spectral data were collected using the 30 $\mathrm{mm}$ lens and under the $500 \mathrm{~W}$ illumination (Set 1, Table 1). An average spectrum from the middle of each sample (region of interest $-\mathrm{ROI}=3 \times 3$ pixels) in the target was extracted. PCDA method was used with the aim to get the lowest error rate on cross-validation results. Validation of the model was based on the percentage of correctly identified samples.

Whatman filter papers (4 layers) were used as the background of the samples. The spectral region 1450-2350 $\mathrm{nm}$ was used and pre-treated using SNV method. LDA was applied on each PC from 1-25 PCs generated from the PCA where the PC number that gave the highest \% correctness on cross-validation was selected.

The highest results were $83.8 \%$ successfully identified samples out of 105 samples used in the calibration with 9 PCs, which proves the ability of the HSI technique for discrimination.

\subsection{Regression Model (Degree of Polymerization)}

A regression model was developed for the prediction of DP values for unknown samples based on the 57 samples from the calibration target that were measured using the viscometric method. DP of cellulose in paper is one of the important characteristics for cellulosic materials that gives an overview of the current material condition.

Similar to the discrimination model, spectral data were collected using the acquisition parameters of Set 1 in Table 1 with Whatman paper background as well. Savitzky-Golay (SG) filter $\left(1^{\text {st }}\right.$ derivative) with different polynomials $(2,3$ and 4) and filtering window sizes from 5-55 points was used to pre-treat the data and the optimal parameters for the model were selected. The model was calculated for each PLS factor from 1-25 and the factor that gave the best validation results based on the root mean square error of the cross validation (small RMSECV) and the Correlation coefficient (high Rcv) was selected.

The optimal calibration model under the described parameters gave an RMSECV $=308$ and $\mathrm{Rcv}=0.73$ using 14 factors, $2^{\text {nd }}$ polynomial and 25-points window size. The model has a potential to be used in collection surveys similar to the models previously developed using the other spectroscopic techniques ${ }^{11,23}$.

\subsection{Evaluation Methodology}

The two developed regression (PLS) and discrimination (PCDA) models for determination of DP and starch presence were used as an evaluation methodology to study the effect of the different measurements and calibration parameters and processing methods such as scanning backgrounds, lenses, ROI size for the average spectra and pre-treatment methods on the accuracy of the results.

Spectralon diffuse reflectance standard target is considered one of the common reference standards used in most of the spectroscopic applications for calibrations due to its flatness and constant reflectivity over the range $250-2500 \mathrm{~nm}^{10,24}$. However, due to the cost of such standards it is difficult to use a standard target of the same size as the scanner stage (A3 size), thus Whatman filter paper No. 1 was seen as an alternative as a pure cellulose material similar to the objects under investigation. The Islamic paper calibration target was scanned with the two backgrounds and without one, then PLS models were calculated for each dataset using the spectral range $1450-2350 \mathrm{~nm}$ to evaluate the effect on the results of the quantitative calibration model. The data were acquired using the $30 \mathrm{~mm}$ lens and under $500 \mathrm{~W}$ illumination (Set 1, Table 1). The different PLS model results are shown in Table 2 comparing the RMSECV and Rcv values. RMSECV values do not change substantially, taking into consideration the typical error of the traditional viscometric method to determine the DP.

On the other hand, using the same spectral datasets, different PCDA models were developed and showed that Spectralon background leads to the most optimal results with $93.3 \%$ accuracy compared to $83.8 \%$ using the Whatman background and $79 \%$ using no background.

It is common practice in the analysis of spectral data and the development of calibration models to use spectral pretreatments methods to help in preparing the data and avoid noise or scatter. However, its selection depends on testing and experience $e^{4,10}$ which may cause variations in the results. Thus, in this paper, different pre-treatment methods were 
evaluated to explore these effects. The same collected spectral datasets that were scanned with the different backgrounds were pre-processed with three different methods; Multiplicative scatter correction (MSC), standard normal variate (SNV) and Savitzky-Golay filter (1st and 2nd derivatives) (SG) then the PLS regression model was calculated for each and also calculated from the RAW data. All results are presented in Table 2 which are showing slight variations between using the different methods.

Table 2. PLS regression model results for determination of DP using HSI data of Islamic Paper target with two different backgrounds and without one. The calculations were done using different pre-treatment methods for the collected spectra in the range $1450-2350 \mathrm{~nm}$ to avoid noisy edges.

\begin{tabular}{|c|l|l|l|l|}
\hline Background & \multicolumn{1}{|c|}{ Pre-treatment } & PLS factors & RMSECV & \multicolumn{1}{c|}{ Rcv } \\
\hline \multirow{5}{*}{ Spectralon } & No pre-treatment & 7 & 330 & 0.66 \\
\cline { 2 - 5 } & MSC & 9 & 310 & 0.71 \\
\cline { 2 - 5 } & SNV & 7 & 314 & 0.70 \\
\cline { 2 - 5 } & $(\mathrm{SG}) 1^{\text {st }}$ derivative & 10 & 286 & 0.75 \\
\cline { 2 - 5 } & $(\mathrm{SG}) 2^{\text {nd }}$ derivative & 10 & 286 & 0.75 \\
\hline \multirow{5}{*}{ Whatman } & No pre-treatment & 10 & 332 & 0.68 \\
\cline { 2 - 5 } & MSC & 8 & 337 & 0.66 \\
\cline { 2 - 5 } & SNV & 8 & 329 & 0.70 \\
\cline { 2 - 5 } & $(\mathrm{SG}) 1^{\text {st }}$ derivative & 14 & 308 & 0.73 \\
\cline { 2 - 5 } & $(\mathrm{SG}) 2^{\text {nd }}$ derivative & 3 & 322 & 0.65 \\
\hline & No pre-treatment & 21 & 292 & 0.75 \\
\cline { 2 - 5 } & MSC & 15 & 308 & 0.72 \\
\cline { 2 - 5 } & SNV & 15 & 316 & 0.71 \\
\cline { 2 - 5 } & $(\mathrm{SG}) 1^{\text {st }}$ derivative & 5 & 300 & 0.71 \\
\cline { 2 - 5 } & $(\mathrm{SG}) 2^{\text {nd }}$ derivative & 9 & 296 & 0.75 \\
\hline
\end{tabular}

Illumination in any imaging system has a great influence on the reliability of the collected data ${ }^{2,25}$. There is always a trade-off between the intensity of illumination and the exposure/integration time needed to achieve the optimal conditions, which additionally depends on the sensitivity of the object under investigation. Therefore, both modes of illumination $(250$ and $500 \mathrm{~W})$ were tested to evaluate their effect of the collected spectra. For the acquisition parameters for each intensity, see Set 1 and Set 2 in Table 1. The difference of the average spectra of the whole target from both scans is showing no variation except for random noise at the edges (Figure 3 - right). A slight peak in the range 1900 $1950 \mathrm{~nm}$ was observed, which could be the effect of changes in the relative humidity from one scan to another (moisture peak) but further investigation is needed. 


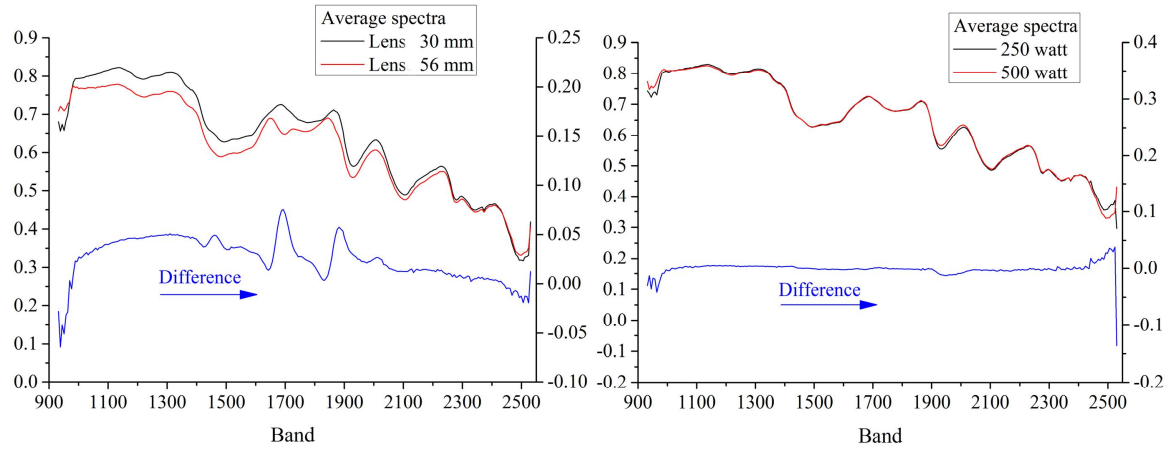

Figure 3. Two average spectra and their difference, collected from the Islamic paper target using HSI scanner using different acquisition parameters; two illumination intensities: 250 and $500 \mathrm{~W}$ (right) and two lenses; 30 and $56 \mathrm{~mm}$ (left) to visualize variability in the measurements.

Spatial resolution (ability to get the spatial details required in an image) is one of the key parameters of an HSI system. It depends on different components in the system such as scanning distance, lens, aperture, detector and electronic ${ }^{25,26}$. Since most of these parameters are fixed in a given setup, a scanning test using the Islamic paper calibration target was conducted using two lenses $(30$ and $56 \mathrm{~mm}$ ) to evaluate the influence on the collected data. Table 1 includes the acquisition parameters used for the two scans (Set 1 and Set 3). Similar to the illumination test, the difference of the average spectra of the target from the two scans was calculated. As seen in Figure 3 (left), there is a noticeable difference between both scans which could affect the analysis results and requires more investigation.

Since a difference in the spectra was observed using different lenses, the PCDA discrimination model was calculated using these datasets to check if this variation in the spectra affects the calibration results. The Whatman paper background was used and scanning was carried out using $500 \mathrm{~W}$ illumination. Table 3 shows the results of the model for each lens.

ROI size could also be a parameter that may affect the results, so this was tested as well using the same spectral datasets of the two lenses. It could be argued that the more pixels we get from each sample square ( $7 \times 7$ pixels) in the target, the more representative the sample will be. Therefore, two different ROI sizes; $3 \times 3$ and $5 \times 5$ pixels from the calibration target scans were tested using the developed PCDA models and the results are given in Table 3.

As shown, both parameters lead to have a slight change in the results indicating its low effect on the calibration accuracy.

Table 3. PCDA model results for identification of starch presence using HSI data of Islamic Paper target collected by two different lenses; 30 and $56 \mathrm{~mm}$. The calculations were done based on an average spectra of two different ROI sizes $(3 \times 3$ and $5 \times 5)$ from each sample in the target. Measurements were collected in the range 1450-2350 nm with Whatman filter paper as background and pre-treated using SNV method.

\begin{tabular}{|l|l|l|l|}
\hline Lens & ROI & PC number & \% Correctness \\
\hline $30 \mathrm{~mm}$ & $3 \times 3$ & 9 & $83.8 \%$ \\
\hline $56 \mathrm{~mm}$ & $3 \times 3$ & 17 & $84.8 \%$ \\
\hline $30 \mathrm{~mm}$ & $5 \times 5$ & 21 & $86.7 \%$ \\
\hline $56 \mathrm{~mm}$ & $5 \times 5$ & 15 & $84.8 \%$ \\
\hline
\end{tabular}

\section{CONCLUSION}

In this paper, hyperspectral imaging (1000-2500 nm) was explored for non-destructive quantitative characterization of Islamic paper focusing on the effect of the use of different acquisition and data analysis parameters on the accuracy of the results. A custom-made calibration target made from 105 samples of Islamic paper from a well-characterized collection was used to enable the development of quantitative calibration models. Different spectral pre-processing and multivariate data analysis methods were applied to the collected spectral data. A discrimination model was developed to establish the presence of starch as an important characteristic of Islamic paper and a PLS regression model was 
developed for quantitative mapping of cellulose DP in paper, which could provide visual cues to the current condition of the object. Both models were used as an evaluation methodology for the different parameters under investigation.

A pushbroom HSI scanner from Gilden Photonics with a spectral resolution of $6.3 \mathrm{~nm}$ using a mirror scanning setup and halogen illumination was used to collect the spectral data. The calibration target was scanned and analyzed with different measurement conditions, acquisition parameters and post-processing methods.

Different scanning backgrounds, lenses and illumination intensities were tested. Also, three pre-treatment methods were explored in addition to different sizes of the extracted average spectrum for each sample in the calibration target. Results based on the evaluation methodology are showing that measurement parameters may have a slight to no influence on the quantitative results. In addition, the methodology was able to give guidelines for the selection of the optimal postprocessing method to be applied to the spectral data.

The research aims to have a significant impact on our understanding of heritage surfaces as spatially resolved quantitative data could improve the interpretation and conservation of historic materials of cellulosic origin.

\section{ACKNOWLEDGEMENTS}

The authors gratefully acknowledge the financial support of the EPSRC Centre for Doctoral Training in Science and Engineering in Arts, Heritage and Archaeology (SEAHA), and the European Union's Horizon 2020 research and innovation programme project "Nanorestart".

\section{REFERENCES}

[1] Strlič, M., and Kolar, J., [Ageing and stabilization of paper], National and university library, Ljubljana, Slovenia (2005).

[2] Fischer, C., and Kakoulli, I., "Multispectral and hyperspectral imaging technologies in conservation: current research and potential applications," Reviews in conservation 7, 3-16 (2006).

[3] Lu, R. and Chen, Y., "Hyperspectral imaging for safety inspection of food and agricultural products," Proceedings of the SPIE, Pathogen Detection and Remediation for Safe Eating, 3544, 121-133 (1999).

[4] Wang, W. and Paliwal, J., "Near-infrared spectroscopy and imaging in food quality and safety," Sensing and Instrumentation for Food Quality and Safety, 1(4), 193-207 (2007).

[5] Liang, H., "Advances in multispectral and hyperspectral imaging for archaeology and art conservation," Applied physics: A: Materials science \& processing, 106, 309-323 (2012).

[6] Dooley, K., Lomax, S., Zeibel, J., Miliani, C., Ricciardi, P., Hoenigswald, A., Loewb, M., and Delaney, J., "Mapping of egg yolk and animal skin glue paint binders in early renaissance paintings using near infrared reflectance imaging spectroscopy," RSC publishing, Analyst, 138(17), 4838-4848 (2013).

[7] Cséfalvayová, L., Strlič, M., Karjalainen, H., "Quantitative NIR chemical imaging in heritage science," Analytical chemistry, 83(13), 5101-5106 (2011).

[8] Lawrence, K., Park, B., Windham, W., and Mao, C., "Calibration of a pushbroom hyperspectral imaging system for agricultural inspection," Transactions of the American Society of Agricultural Engineers-ASAE, 46(2), 513-521 (2003).

[9] Elmasry, G. and Sun, D., "Principles of hyperspectral imaging technology," [Hyperspectral Imaging for Food Quality Analysis and control], Chapter 1, Elsevier, 3-43 (2010).

[10] Yao, H. and Lewis, D., "Spectral preprocessing and calibration techniques," [Hyperspectral Imaging for Food Quality Analysis and Control], Chapter 2, Elsevier, 45-78 (2010).

[11] Mahgoub, H., Bardon, T., Lichtblau, D., Fearn, T., and Strlič, M., "Material properties of Islamic paper," Heritage Science, 4(34), (2016).

[12] Isenberg, I., [Pulp and paper microscopy], Institute of Paper Chemistry, 3rd edition, Wisconsin (1967).

[13] Baker, D., "Arab papermaking," The paper conservator, 15, 28-35 (1991).

[14] Evans, R., and Wallis, A., "Comparison of cellulose molecular weights determined by high performance size exclusion chromatography and viscometry," 4th international symposium on wood and pulping chemistry, Paris, 1, 201-205 (1987).

[15]BS-ISO-5351, "Pulps - determination of limiting viscosity number in cupriethylenediamine (CED) solution," 2nd edition, British Standardization Institute-BSI, UK, (2010). 
[16] Manley, M., "Near-infrared spectroscopy and hyperspectral imaging: non-destructive analysis of biological materials," Chemical Society Reviews, Royal society of chemistry- RSC, 43, 8200-8214 (2014).

[17] Blanco, M., and Villarroya, I., "NIR spectroscopy: a rapid-response analytical tool," Trac. trends in analytical chemistry, 21, 240-250 (2002).

[18] Naes, T., Isaksson, T., Fearn, T., and Davies, T., [A user Friendly guide to Multivariate Calibration and Classification], NIR Publications, Chichester, UK, (2002).

[19] Stuart, B., "Molecular spectroscopy," [Analytical techniques in materials conservation], Chapter 4, John Wiley $\&$ sons, Chichester, England, (2007).

[20] Miller, J., and Miller, J., [Statistics and chemometrics for analytical chemistry], $6^{\text {th }}$ edition, Prentice Hall $/$ Pearson, England, (2010).

[21] Brereton, R., "Chemometrics for pattern recognition," John Wiley \& son, England, (2009).

[22] Brereton, R., and Lloyd, G., "Partial least squares discriminant analysis: taking the magic away," Journal of chemometrics, special issue - tutorial, 28(4), 213-225 (2014).

[23] Trafela, T., Strlič, M., Kolar, J., Lichtblau, D., Anders, M., Pucko-Mencigar, D., and Pihlar, B., "Nondestructive analysis and dating of historical paper based on IR spectroscopy and chemometric data evaluation," Analytical Chemistry, 79, 6319-6323 (2007).

[24]Kubik, M., "Hyperspectral imaging: A new technique for the non-invasive study of artworks," [Physical techniques in the study of art, archaeology and cultural heritage], Chapter 5, 2, 199-259 (2007).

[25] Qin, J., "Hyperspectral imaging instruments," [Hyperspectral Imaging for Food Quality Analysis and Control], Chapter 5, Academic Press, Elsevier, 127-172 (2010).

[26] Macdonald, L., "The limits of resolution," Proceeding eva'10 international conference on electronic visualization and the arts, British Computer Society-BCS learning \& development ltd, UK, 149-156 (2010). 